\title{
WAEX: Web Accessibility Evaluator in a single XSLT file
}

\author{
Vicente Luque Centeno, Carlos Delgado Kloos \\ Carlos III University of Madrid \\ Email: $\{$ vlc,cdk $\} @$ it.uc3m.es
}

\author{
Martin Gaedke, Martin Nussbaumer \\ University of Karlsruhe \\ Email: \{gaedke,nussbaumer\}@tm.uni-karlsruhe.de
}

\begin{abstract}
Web accessibility rules, i.e., the conditions to be met by Web sites in order to be considered as accessible for all, can be (partially) checked automatically in many different ways. Monolitic applications built on top of procedural programming languages like $\mathbf{C}$, Java, Perl or PHP have been developed during the last years. These applications usually provide their own interpretation of the $\mathrm{W3C}$ guidelines, so it is easy to obtain different evaluation results when different evaluation tools are applied to a common sample page.
\end{abstract}

Since most accessibility rules are involved with the markup of Web sites and XSLT can be applied to any selectable-by-condition markup, we have developed WAEX as a Web Accessibility Evaluator in a single XSLT file. Such XSLT file contains 50+ singular accessibility rules and XHTML-specific conditions that are in the prose of the XHTML specification because they could not be included in the grammar specification (the DTD or the XML Schemas). This approach of using XSLT as a declarative repository of accessibility rules has lead to a portable and reusable style sheet that generates rather complete (if compared with already existing evaluation tools) accessibility reports for any Web page.

\section{INTRODUCTION}

WAI (Web Accessibility Initiative)'s WCAG (Web Content Accessibility Guidelines) 1.0 [1] from W3C has become an important reference for Web accessibility in the Web community. It has been accepted as a set of guidelines that improve accessibility and eliminate barriers on Web sites. The lack of accessibility affects a large amount of people (between 10\% and 20\%) that frequently find important barriers when trying to navigate through today's Web sites. Barriers reduce accessibility not only for people with some sort of personal disability (vision or hearing impairment, mobility problems to manage a keyboard or a mouse, or cognitive and neurological problems). Accessibility is also a major step towards device independent Web design, allowing Web interoperability to be independent from devices, browsers or operating systems. Web accessibility allows having cheaper Web site maintenance and a wider target public. Some governments are also requiring that some Web sites become accessible.

The set of the 65 WCAG's checkpoints that accessible documents have to pass is a very heterogeneous set of constraints, which are difficult to evaluate and repair. Both WCAG 1.0 [1] and the HTML Techniques for the new WCAG 2.0 draft [2] specifications are written at a high abstraction level, which is frequently quite open to subjective interpretation (as too long texts or too many elements), including implicit conditions or, simply, containing constraints whose detection cannot be automated.

There are several tools nowadays which can help us to detect accessibility barriers on Web sites. Watchfire[9], Tawdis [10] or HERA [11] are only a few of them. It is well known that all these tools require a person to supervise and complete the results of the evaluation tools because a lot of rules are relayed on manual checks by the user. However, we still have the problem of different particular interpretations on each tool. This is due to the fact that these tool's authors have had different subjective interpretations of the W3C's guidelines, as depicted at [17]. As a result, we can easily find several tools reporting different evaluation results for a same sample page. Even further, it is difficult indeed to find two evaluation tools that evaluate the same conditions.

One of the main reasons for this heterogeneity is due to the way these tools have been implemented. Since they have been mostly implemented using procedural programming languages, the conditions being checked are unclear for people using them for Web site evaluation. Our approach to solve that problem consists on providing declarative rules, readable and reusable by anyone, even for those with little programming background.

Our approach lead us to build WAEX [15]: a Web 
Accessibility Evaluator in a single XSLT file. Because of its XSLT nature, it can only be applied to well formed HTML pages. Fortunately, HTML reparation software like Tidy [13] and the HTML parser of libxml [14] allows us to apply WAEX to any Web page.

The rest of this paper depicts the most important WAEX's rules, and is organized as follows: Section II deals with accessibility conditions already expressed formally in the XHTML grammar (the DTD or XML Schema) and compares them with the corresponding XSLT templates in WAEX. Section III deals with those accessibility conditions expressible in a XHTML grammar, but not expressed indeed in a DTD or Schema. Section IV covers accessibility conditions not expressible in a XHTML grammar. Section V contains some conclusions and future work.

\section{ACCESSIBILITY CONDITIONS ALREADY EXPRESSED IN THE XHTML GRAMMAR}

One of the most important accessibility issues involve validation against a public grammar. In fact, the XHTML grammar already represents some well known accessibility checkpoints. Specific rules in the DTD or XML Schema of XHTML already require that specific mandatory markup properly appears in XHTML documents. For instance, table I contains rules that state that every image must always have an alt attribute. While both the DTD, the XML Schema declare the alt attribute as required for every image, XSLT can be used to spot any image having no such alt attribute as a barrier. Such a rule in WAEX may easily report the absence of this important attribute for accessibility (the alt attribute), because all these rules state that every image with no alt attribute would be considered as faulty since users that cannot see the image can't read a textual alternative either. ${ }^{12}$

Mandatory elements can also be required by a grammar. For instance, table II contains rules to assert that every document has a title (an essential element for the user to differentiate Web pages) and that it must be directly inside the head element. In fact, they also assert that only a single title must be present. Having none or multiple titles is considered as an error. While both

\footnotetext{
${ }^{1} \mathrm{~A}$ similar set of expressions can be used to state that the alt attribute is also required for every area element.

${ }^{2}$ Ornamental images containing no information, should have an empty string as the textual alternative, but this attribute must be present anyhow according to WCAG. The fact that the textual alternative is an adequate alternative for the image is another checkpoint which is outside of our scope.
}

the DTD and XML Schema express that condition in a regular expression, XSLT may be used to assert that the title must exist and it must be unique.

Validation is also useful for detecting forbidden or deprecated markup within a document. It is important to avoid deprecated markup in order to properly separate structure from presentation. For instance, bullets in list items should be specified only by a CSS (Cascading Style Sheet), instead of using the list's type attribute, because they are considered as a presentational feature. Even though such an attribute is allowed in XHTML Transitional [2], it has been eliminated on XHTML Strict and successive XHTML versions because it has been deprecated. Table III states that lists should no longer have any such type attribute. ${ }^{3}$ The XSLT expression provided in table III explicitly forbids this, while DTD and XML Schema declare that implicitly.

Deprecated and forbidden elements can also be detected if a modern XHTML grammar is being used for validation. Deprecated elements like b (bold), i (italic), tt (tele-type), center or font, as well as forbidden elements not in the HTML specification like blink or marquee, can be spotted (as presentational markup that should be left out in favour of CSS) by either a grammar which does not recognize them as valid elements or with an XSLT rule like the one at table IV.

\section{ACCESSIBILITY CONDITIONS EXPRESSIBLE (BUT NOT EXPRESSED) IN A XHTML GRAMMAR}

XHTML is not a single language. Since its birth, it has had several versions, starting from Transitional and Strict [2], launching XHTML Basic [4] and ending by XHTML 1.1 [3]. Those different languages have different restrictions, some of them had been previously declared in the 1999's WCAG [1]. For example, the alt attribute is mandatory since XHTML Transitional 1.0. Deprecated elements like font or center were removed in XHTML Strict 1.0. XHTML 1.1 introduced some new rules and removed some deprecated features from XHTML Strict 1.0. XHTML Basic does not allow frames, plugins, scripts, both server or client side image maps or nested tables for layout, thus implying that those WCAG rules referring to these elements simply can't be broken. XHTML Basic was designed as the minimum subset of XHTML tags and attributes that all browsers should understand, especially oriented to small

\footnotetext{
${ }^{3}$ Other deprecated attributes like bgcolor or forbidden ones like bordercolor are also implicitly forbidden by grammar.
} 


\begin{tabular}{|l|l|}
\hline & Expression \\
\hline DTD & $<$ !ATTLIST img alt CDATA \#REQUIRED $>$ \\
\hline XML Schema & $<$ xs:element name="img" $><$ xs:complexType $>$ \\
& $<$ xs:attribute name="alt" use="required" \\
& type="xs:string"/ $>$ \\
& $<$ /xs:complexType $></$ xs:element $>$ \\
\hline XSLT & $<$ xsl:template match="//img[not(@alt)]" $>$ \\
& $<$ xsl:message $>$ Images with no alt attribute $</$ xsl:message $>$ \\
& $</$ xsl:template $>$ \\
\hline
\end{tabular}

\section{TABLE I}

IMAGES WITHOUT ALTERNATIVE TEXT. THE img's alt ATTRIBUTE IS MANDATORY.

\begin{tabular}{|c|c|}
\hline & Expression \\
\hline DTD & $\begin{array}{l}<\text { !ELEMENT head (\%head.misc; } \\
\quad((\text { title, \%head.misc;, (base, \%head.misc;)?) } \\
\quad \text { (base, \%head.misc;, (title, \%head.misc;))))> }\end{array}$ \\
\hline XML Schema & $\begin{array}{l}\text { <xs:element name="head" }> \\
\text { <xs:complexType }><\text { xs:sequence }> \\
\text { <xs:group ref="head.misc"/ }> \\
\text { <xs:choice }><\text { xs:sequence }> \\
\text { <xs:element ref="title"/ }><\text { xs:group ref="head.misc"/ }> \\
\text { <xs:sequence minOccurs="0" }> \\
\text { <xs:element ref="base"/ }><\text { xs:group ref="head.misc"/ }> \\
\text { </xs:sequence }> \\
\text { </xs:sequence }><\text { xs:sequence }> \\
\text { <xs:element ref="base"/ }><\text { xs:group ref="head.misc"/ }> \\
\text { <xs:element ref="title"/ }><\text { xs:group ref="head.misc"/ }> \\
</ \text { xs:sequence }></ \text { xs:choice }> \\
<\text { /xs:sequence }></ \text { xs:complexType }> \\
</ \text { xs:element }>\end{array}$ \\
\hline XSLT & $\begin{array}{l}<\text { xsl:template match="/html[count }(. / \text { head/title }) !=1] "> \\
\quad<\text { xsl:message }>\text { Document without a unique title }</ \text { xsl:message }> \\
<\text { /xsl:template }>\end{array}$ \\
\hline
\end{tabular}

Document Without A UniQue TITLE. Document's TITLE IS MANDATORY.

\begin{tabular}{|c|c|}
\hline & Expression \\
\hline DTD & $\begin{array}{l}\text { Though declarations such as } \\
<\text { !ATTLIST ul type "(disc } \mid \text { square } \mid \text { circle)" \#IMPLIED }> \\
\text { might exist (in older XHTML versions), such attributes should not be used }\end{array}$ \\
\hline XML Schema & $\begin{array}{l}\text { Though declarations such as } \\
<\mathrm{xs} \text { :attribute name="type"/> inside } \\
<\mathrm{xs} \text { :element name="ul"/> might exist (in older XHTML versions), such } \\
\text { attributes should not be used }\end{array}$ \\
\hline XSLT & $\begin{array}{l}<\text { xsl:template match="(//ul } \mid / / \text { ol })[@ \text { type]" }> \\
<\text { xsl:message }>\text { List item's bullet should be specified by } \\
\text { CSS }</ \text { xsl:message }> \\
</ \text { xsl:template }>\end{array}$ \\
\hline
\end{tabular}

TABLE III

LIST ITEM'S BULLET SHOULD BE SPECIFIED BY CSS 


\begin{tabular}{|c|c|}
\hline & Expression \\
\hline DTD & $\begin{array}{l}\text { No such }<\text { !ELEMENT b ...> nor } \\
<! \text { ELEMENT i ...> ... (in new XHTML versions) }\end{array}$ \\
\hline XML Schema & $\begin{array}{l}\text { No such <xs:element name="b"/> nor } \\
<\text { xs:element name="i"/> .. (in new XHTML versions) }\end{array}$ \\
\hline XSLT & $\begin{array}{l}\text { <xsl:template match="//b }|/ / / \mathrm{i}| / / \text { tt } \mid \text { //font } \mid \text { //center } \mid / / \text { blink" }> \\
\quad<\text { xsl:message }>\text { Deprecated elements no longer in use }</ \text { xsl:message }> \\
<\text { /xsl:template }>\end{array}$ \\
\hline
\end{tabular}

DEPRECATED ELEMENTS NO LONGER IN USE

devices with smaller capabilities. Its usage involves that presentation completely relies on CSS.

The refinement process started by XHTML 1.0 and ended by XHTML 1.1 has already included rules expressible in a grammar into successive DTDs and/or XML Schemas. However, some refinements still remain uncompleted. For example, WCAG 1.0 states that all frames should have a mandatory title attribute. Even though this rule is very similar to the one exposed at table I, the XHTML grammar still defines this attribute as optional, instead of required. For this reason, rules from table $\mathrm{V}$ are required, unless we locally redefine this rule in our own DTD or XML Schema. ${ }^{4}$

Table VI contains a summary of XHTML prohibitions stated in the prose of XHTML specification. Such restrictions could be easily expressed formally in the XHTML DTD. However, they were not formalized by any rule.

The best of having checkpoints guaranteed by a grammar is that they are very cheap and easy to detect: just choosing a modern grammar to validate documents against to, and using an existing XML-ized validator like [8], may easily spot where barriers belonging to this category appear within a document.

\section{ACCESSIBILITY CONDITIONS NOT EXPRESSIBLE IN A XHTML GRAMMAR}

There are some accessibility conditions that are not expressible indeed in a DTD or XML Schema. Many tools have their own internal implementation of these conditions hard-coded within lines of a program coded in an procedural programming language like Java, C or PHP. This might lead to complex algorithms which (usually) implement something different from the desired checkpoint. Approaches like [12] have tried to represent such

\footnotetext{
${ }^{4}$ A similar approach (DTD or Schema redefinition) could be taken for the usage of the noframes element, because the public DTD does not properly require such element even though it is required to be present as an alternative for frames for browsers that can't support frames.
}

rules in a set of declarative expressions declared in selfdeveloped XML files that represent rules which can only be interpreted by a self-developed ad-hoc program. Using already existing software was the aim of the XQuerybased WCAG formalization approach [16]. However, though the XQuery language [6] is quite compact, it is still a draft having little implementation support. A more accurate and also cheaper implementation of checkpoint evaluation can be achieved when using other already known constraint languages for XML, like XSLT. This approach implies cheaper evaluation because no specific software needs to be developed indeed, and there are specific programs already supporting this language. As a result, we have a XSLT-based declarative ruleset which is smaller, more reusable and easier to understand and homogeneize than the equivalent procedural routines.

As a starting example, we will begin with attributes that enhance accessibility, but whose presence is required only under certain circumstances. For instance, table VII states that every image input, (i.e., input form fields whose type attribute is image) requires the alt attribute (as any other image). It would not be nice to declare the alt attribute as mandatory for every input element, because input form fields having a type attribute different of image don't really expect such alt attribute. Conditions that indicate whether some markup is mandatory or not, are not expressible in DTDs or XML Schemas.

In fact, conditions that involve some comparison or calculation over the document's content, are not expressible in DTDs or XML Schemas. Other accessibilityrelated attributes are also required in other elements under other conditions as well. Table VIII contains such required attributes, their container elements and their conditions to be mandatory. They are summarized, but they can be used just like the expressions in table VII.

Not only the presence or absence of markup determines accessibility. The conditions themselves may become an evaluation result. For instance, Web acces- 


\begin{tabular}{|c|c|}
\hline & Expression \\
\hline DTD & $\begin{array}{l}<! \text { ATTLIST frame title CDATA \#REQUIRED }> \\
\text { However, it is defined as \#IMPLIED (not required) }\end{array}$ \\
\hline XML Schema & $\begin{array}{l}<\text { xs:element name="frame" }><\text { xs:complexType }> \\
<\text { xs:attribute name="title" use="required" } \\
\text { type="xs:string"/ }> \\
</ \text { xs:complexType }></ \text { xs:element }> \\
\text { However, it is defined as optional (not required) }\end{array}$ \\
\hline XSLT & $\begin{array}{l}<\text { xsl:template match="//frame[not }(@ \text { title })] "> \\
\quad<\text { xsl:message }>\text { Frames without description. }<\text { /xsl:message }> \\
<\text { /xsl:template }>\end{array}$ \\
\hline
\end{tabular}

TABLE V

FRAMES WITHOUT DESCRIPTION. THE frame's tit le ATTRIBUTE IS MANDATORY.

\begin{tabular}{|l|l|l|}
\hline $\begin{array}{l}\text { Container el- } \\
\text { ement }\end{array}$ & Forbidden contents & $\begin{array}{l}\text { Condition to be forbid- } \\
\text { den }\end{array}$ \\
\hline pre & img, object, big, small, sub, sup & Always forbidden \\
\hline a, label & a, area, label, input, select, textarea, button & Always forbidden \\
\hline form & form & Always forbidden \\
\hline table & table & In XHTML Basic [4] \\
\hline ins & Any block-type element & $\begin{array}{l}\text { If the ins element is in- } \\
\text { side an inline-type ele- } \\
\text { ment }\end{array}$ \\
\hline
\end{tabular}

RESTRICTIONS IN XHTML SPECIFICATION (PROSE) NOT EXPRESSED IN THE DTD

\begin{tabular}{|c|c|}
\hline & Expression \\
\hline DTD & Not feasible \\
\hline XML Schema & Not feasible \\
\hline XSLT & $\begin{array}{l}<\text { xsl:template match="//input[@ } @ \text { type='image'][not(@alt)]" }> \\
<\text { xsl:message }>\text { Image input with no alt }</ \text { xsl:message }> \\
<\text { /xsl:template }>\end{array}$ \\
\hline
\end{tabular}

\section{TABLE VII}

IMAGE INPUTS WITHOUT ALTERNATIVE TEXT. THE alt ATTRIBUTE IS MANDATORY.

\begin{tabular}{|l|l|l|}
\hline $\begin{array}{l}\text { Container el- } \\
\text { ement }\end{array}$ & Attribute & Condition to be mandatory \\
\hline img,area & alt & Always \\
\hline frame & title & Always \\
\hline input & alt & If input's type is "image" \\
\hline img & longdesc & If image's alt attribute is not enough \\
\hline html & lang & If document's xml: lang is missing \\
\hline frame & longdesc & If frame's tit le attribute is not enough \\
\hline th & abbr & If table header's text is too long \\
\hline input & value & If form field collects editable text \\
\hline table & summary & $\begin{array}{l}\text { If table contains tabular data, i.e, it is not a } \\
\text { layout-only table }\end{array}$ \\
\hline abbr,acronym & title & If definition not previously defined \\
\hline
\end{tabular}

ATTRIBUTES CONDITIONALLY REQUIRED. 
sibility checkpoints state that both keyboard and mouse should behave similarly in order to have some device independence properties. This involves that any JavaScript routine triggered by a mouse event should also be triggered as well by the corresponding keyboard event, and vice-versa. Table IX contains an XSLT template checking whether the onmouseup event is being used for other purpose than the onkeyup event. A more complete rule should also check the same behaviour for other events. ${ }^{5}$

Not only conditionally mandatory attributes can be required, but also conditionally mandatory elements as well. For instance, multimedia elements like plugins, applets or videos should have alternative contents for those users that cannot interact with them properly. These alternative contents must be placed directly inside the object element itself (the object tag acts as a container of its alternative), as stated in table X. Take notice that param elements are not considered as alternative contents.

Thus, any object element should have some other alternative element inside if it does not have a readable alternative text. Other conditionally required elements are represented in table XI. For instance, some accessibility checkpoints state that fieldset elements should be present in order to make manageable groups of form fields. When the number of options in a combo box (select element) is high, it is a good idea to group them with the optgroup element. The expressions from table XI are summarized, but they can be used just like the expressions in table $\mathrm{X}$.

Some other important accessibility rules don't really involve existence or absence, but misusage of HTML markup. These rules state that some HTML attributes should not be improperly used. For example, it is a very bad practice to trigger JavaScript routines from the href attribute of active elements like links or client-side map areas. It is much better to use event-focused attributes like onclick for that purpose, and leave the href attribute usable for users that have browsers with no JavaScript support. The XSLT template from table XII detects this bad practice.

Other bad practices involving typically misused attributes are collected in table XIII. As we can see, it is bad practice to use client side auto-refresh or

\footnotetext{
${ }^{5}$ Also for onmousedown, onkeydown, onkeypress, onmouseover, onmouseout, onmousemove, ondblclick, ...
}

auto-redirect, unadvised emerging windows, improper keyboard short-cuts or unclear tabulation orders.

However, the expressive power of XSLT becomes clearer when we leave out these previous simple conditions involving the relationship between elements and their contents and we start looking at relationships among elements placed anywhere in the document. For instance, headers ( $\mathrm{h} 1-\mathrm{h} 6$ elements) in a document should be properly used. This involves not skipping header labels. As stated at [1], "h2 elements should follow h1 elements, h3 elements should follow h2 elements, etc. Content developers should not skip levels (e.g., h1 directly to h3)". Table XIV contains a XSLT template that spots those headers that skip levels. The preceding XPath axis is used here to determine if the current header is used accordingly to the immediately preceding header in the document. These constraints between one element and its preceding element cannot be expressed with a DTD or XML Schema, but they can fit easily in a XSLT template. ${ }^{6}$

Relationships among non-similar elements can be addressed with constraint languages as well. For example, each client-side map area should have a redundant link (somewhere in the document) for those users that cannot use client-side maps. This implies that, for every area element, at least one link in the same document should share the same destination (i.e. point to the same href). In other words, some link in the document should replicate the href attribute for each area. As inferred from table XV, we have to distinguish between the area element itself and the link before comparing their href attributes. In XSLT, we can use the $<$ xsl:variable $>$ element to reference the area element when the context node has been changed. The XSLT expression from table XV states that any area whose href attribute is not being replicated by a link somewhere else in the document will be spotted as an accessibility barrier.

Expressions involving more than one variable or following internal references can also be expressed with XSLT. This is the case for the relationship between form fields and their label elements. According to accessibility good practices, all non-hidden form fields should have a description stating the purpose of the form field (or what kind of data is expected to be entered).

\footnotetext{
${ }^{6}$ For reducing verbosity, they are presented all together in a singular XSLT template. They might have been written in a template for each header, but we wanted to reduce verbosity and maintain singular templates only.
} 


\begin{tabular}{|l|l|}
\hline ETD & Expression \\
\hline XML Schema & Not feasible \\
\hline XSLT & $\begin{array}{l}<\text { xsl:template match="//*[@ onmouseup or @ onkeyup] } \\
{[\text { not }(@ \text { onmouseup=@ onkeyup)]" }>}\end{array}$ \\
& $\begin{array}{c}<\text { xsl:message }>\text { Device dependence. Both keyboard and mouse should be- } \\
\text { have similarly. }</ \text { xsl:message }> \\
</ \text { xsl:template }>\end{array}$ \\
\hline
\end{tabular}

DEVICE DEPENDENCE. BOTH KEYBOARD AND MOUSE SHOULD BEHAVE SIMILARLY.

\begin{tabular}{|c|c|}
\hline & Expression \\
\hline DTD & Not feasible \\
\hline XML Schema & Not feasible \\
\hline XSLT & $\begin{array}{l}\text { <xsl:template match="//object[normalize-space }(.)="] \\
\quad[\operatorname{not}(*[\text { name }() !=' \text { param'] })] "> \\
<\text { xsl:message }>\text { Multimedia objects without alternative. The alternative in- } \\
\text { side is mandatory. }</ \text { xsl:message }> \\
</ \text { xsl:template }>\end{array}$ \\
\hline
\end{tabular}

Multimedia obJects Without ALTERnATIVE. The ALTERNATIVE INSIDE IS MANDATORY.

\begin{tabular}{|l|l|l|}
\hline $\begin{array}{l}\text { Container el- } \\
\text { ement }\end{array}$ & Required element & Condition to be mandatory \\
\hline head & title & Always \\
\hline html & head & Always \\
\hline $\begin{array}{l}\text { frameset, } \\
\text { iframe }\end{array}$ & noframes & Always \\
\hline object & some alternative inside & If object does not contain alternative text \\
\hline form & fieldset & If too many form fields \\
\hline select & optgroup & If too many options \\
\hline table & caption & $\begin{array}{l}\text { If table contains tabular data, i.e, it is not a } \\
\text { layout-only table }\end{array}$ \\
\hline
\end{tabular}

ELEMENTS CONDITIONALLY REQUIRED

\begin{tabular}{|c|c|}
\hline & Expression \\
\hline DTD & Not feasible \\
\hline XML Schema & Not feasible \\
\hline XSLT & 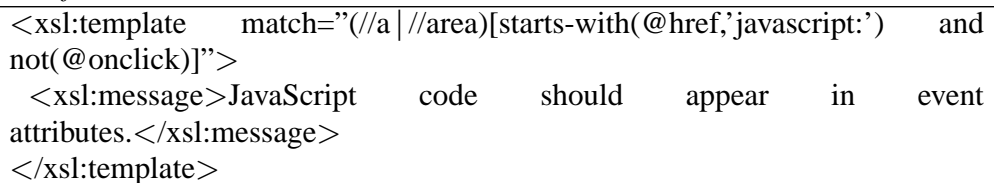 \\
\hline
\end{tabular}

NOT ONLY JAVASCRIPT-BASED NAVIGATION. JAVASCRIPT CODE SHOULD APPEAR IN EVENT ATTRIBUTES.

\begin{tabular}{|l|l|l|}
\hline $\begin{array}{l}\text { Container el- } \\
\text { ement }\end{array}$ & $\begin{array}{l}\text { Misused } \\
\text { attribute }\end{array}$ & Misusage condition \\
\hline a, area & href & $\begin{array}{l}\text { JavaScript present at href attribute, instead of at } \\
\text { onclick attribute }\end{array}$ \\
\hline meta & http-equiv & Badly used for auto-refresh or auto-redirect \\
\hline input & alt & If input's type is not "image" \\
\hline$*($ any tag $)$ & alt & Alt attribute equals src attribute \\
\hline$*($ any tag $)$ & target & Badly used for emerging windows \\
\hline$*($ any tag $)$ & tabindex & Not a set of unique consecutive numbers \\
\hline$*($ any tag $)$ & accesskey & Not a unique character \\
\hline
\end{tabular}

\section{TABLE XIII}

ATTRIBUTES TYPICALLY MISUSED 


\begin{tabular}{|c|c|}
\hline & Expression \\
\hline DTD & Not feasible \\
\hline XML Schema & Not feasible \\
\hline XSLT & $\begin{array}{l}\text { <xsl:template match="//h6[not(preceding::*[self::h1 or self::h2 or self::h3 } \\
\text { or self::h4 or self::h5 or self::h6][1][self::h6 or self::h5])] | } \\
\text { //h5[not(preceding::*[self::h1 or self::h2 or self::h3 or self::h4 or self::h5 } \\
\text { or self::h6][1][self::h6 or self::h5 or self::h4])] | } \\
\text { //h4[not(preceding::*[self::h1 or self::h2 or self::h3 or self::h4 or self::h5 } \\
\text { or self::h6][1][self::h6 or self::h5 or self::h4 or self::h3])] | } \\
\quad / / h 3[\text { not(preceding::*[self::h1 or self::h2 or self::h3 or self::h4 or self::h5 } \\
\text { or self::h6][1][self::h6 or self::h5 or self::h4 or self::h3 or self::h2])] | } \\
\text { //h2[not(preceding::*[self::h1 or self::h2 or self::h3 or self::h4 or self::h5 } \\
\text { or self::h6][1])]"> } \\
\quad<\text { xsl:message>Improper headers }</ \text { xsl:message }> \\
</ \text { xsl:template> }\end{array}$ \\
\hline
\end{tabular}

TABLE XIV

PROPERLY USED HEADERS

\begin{tabular}{|l|l|}
\hline & Expression \\
\hline DTD & Not feasible \\
\hline XML Schema & Not feasible \\
\hline XSLT & $<$ xsl:template match="//area" $>$ \\
& $<$ xsl:variable name="area" select="." / $>$ \\
& $<$ xsl:if test="not(//a[@ @ref = \$area/@ $\mathrm{href}]$ ") $>$ \\
& $<$ xsl:message $>$ Missing redundant area's link $</$ xsl:message $>$ \\
& $</$ xsl:if $>$ \\
& $</$ xsl:template $>$ \\
\hline
\end{tabular}

TABLE XV

A REDUNDANT LINK IS REQUIRED FOR EACH CLIENT-SIDE MAP AREA.

This description should either be in its title attribute or in a label tag referring to it. The XSLT template from table XVI establishes that for any form field that has no title attribute, a label referring to the form field must be present.

Blind people usually find a lot of barriers within a document. They usually read Web pages with screen readers, so they usually navigate through the list of links in a document in order to navigate faster. Thus, it is important that the link's contents can be easily understood when read out of context. Links saying the same words (like "click here" or "read more") and pointing to different targets make navigation difficult. We also must consider that blind users are non-case sensitive when they use screen readers. For that reason, it is a bad practice if two links pointing to different targets share the same pronounceable text. The pronounceable text of a link is a mixture of its text contents, their tit le attribute (which provides extra information) and the alt attributes of all the images inside the link. Although other features like punctuation signs have not been considered in the XSLT expression from table XVII, we can use them to detect similar links pointing to different targets.

\section{CONCLUSiOnS AND FUTURE WORK}

We needed to express rules or templates easy to understand that could be applied to any Web page easily and transparently, i.e., that users could easily understand how to comply with them. We needed that such rules could be reusable and easy to modify. We found that XSLT [7] could be used to express such templates. By applying a self-developed XSLT file, we could generate accessibility reports directly from any Web page.

Soon, we became aware of the expressing power of this approach. We became aware that we could easily express conditions from already built Web Accessibility evaluators, no matter if they were simple or complex in a fine and clean way. We also realized that we could express conditions not previously being checked. For instance, we included conditions from the XHTML specification not previously formalized in the corresponding DTD or Schema.

We have dealt with subjective conditions using the approach of having some customizable parameters in the XSLT file. This way, people might customize some values of the XSLT according to their own needs.

We would have liked to find the required expressing power in DTDs or XML Schema. If so, we would have 


\begin{tabular}{|c|c|}
\hline & Expression \\
\hline DTD & Not feasible \\
\hline XML Schema & Not feasible \\
\hline XSLT & 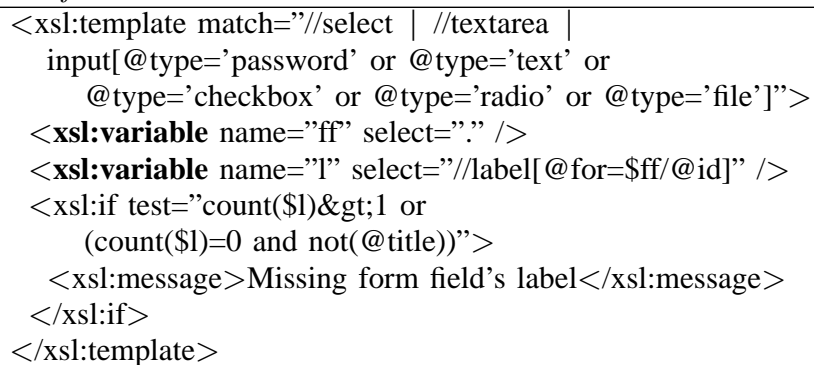 \\
\hline
\end{tabular}

TABLE XVI

A LABEL IS REQUIRED FOR EACH FORM FIELD

\begin{tabular}{|c|c|}
\hline & Expression \\
\hline DTD & Not feasible \\
\hline XML Schema & Not feasible \\
\hline XSLT & 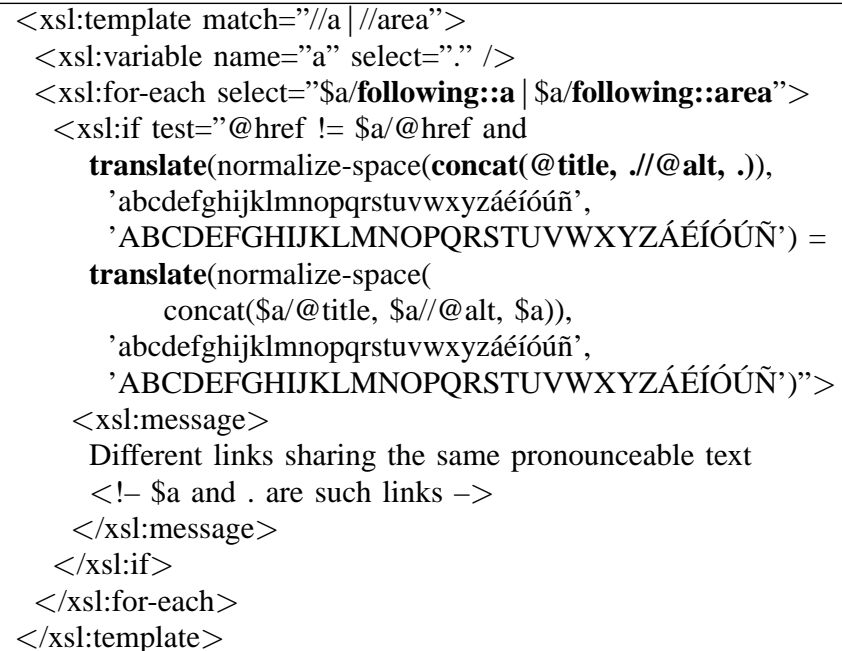 \\
\hline
\end{tabular}

TABLE XVII

AVOID SIMILAR LINKS POINTING TO DIFFERENT TARGETS.

written our conditions in such a grammar (and WAEX would probably be an enhanced DTD or Schema). However, we found that DTDs and XML Schemas don't have the required expressivity, so that's why we used XSLT.

We are planning to adapt WAEX to generate EARL [5] reports as well.

\section{ACKNOWLEDGEMENTS}

We gratefully acknowledge support from the MOSAIC TSI-2005-08225-C07 and INFOFLEX TIC2003-07208 projects of the "Ministerio de Educación y Ciencia" (Spanish ministry). The second author also gratefully acknowledges support from his University and the "Secretaría de Estado de Universidades e Investigación del Ministerio de Educación y Ciencia" of Spain for his sabbatical stay at MIT during the academic year 2005/06, and to the MIT for hosting him during this year in an inspiring environment.

\section{REFERENCES}

[1] W3C Web Content Accessibility Guidelines 1.0 (Recommendation, May 1999) www. w3. org/TR/WCAG10

[2] W3C XHTML 1.0 ${ }^{\text {TM }} 1.0$ - The Extensible HyperText Markup Language (Second Edition), A Reformulation of HTML 4 in XML 1.0, W3C Recommendation 26 January 2000, revised 1 August 2002 www.w3.org/TR/xhtml1

[3] W3C XHTML $1.1{ }^{T M} 1.1$ - Module-based XHTML W3C Recommendation 31 May 2001 www.w3.org/TR/xhtml11

[4] W3C XHTML Basic W3C Recommendation 19 December 2000 www.w3.org/TR/xhtml-basic

[5] W3C Evaluation and Report Language (EARL) 1.0 Guide Editors' Draft 14 February 2006 WWW . w3 . org/TR/EARL10 
[6] W3C XQuery 1.0: An XML Query Language W3C Working Draft 29 October 2004 www.w3.org/TR/xquery

[7] W3C XSL Transformations (XSLT) Version 1.0 W3C Recommendation 16 November 1999 www.w3.org/TR/xslt

[8] W3C Markup Validation Service validator.w3.org

[9] Watchfire WebXACT Accessibility tool webxact.watchfire.com

[10] CEAPAT, Fundación CTIC, Spanish Ministry of Employment and Social Affairs (IMSERSO) Online Web accessibility test www. tawdis. net

[11] Fundación SIDAR Accessibility testing with Style www.sidar.org/hera

[12] Jean Vanderdonckt, Abdo Beirekdar, Monique NoirhommeFraiture Automated Evaluation of Web Usability and Accessibility by Guideline Review 4th Web Engineering International Conference (ICWE 2004), Munich

[13] Sourceforge HTML parser and pretty printer in Java jtidy.sourceforge.net

[14] Daniel Veillard The XML C parser and toolkit of Gnome www. xmlsoft.org

[15] Vicente Luque Centeno WAEX: Web Accessibility Evaluator in a single XSLT file www.it.uc $3 \mathrm{~m}$.es/vlc/waex.html

[16] Vicente Luque Centeno, Carlos Delgado Kloos, Martin Gaedke, Martin Nussbaumer WCAG Formalization with W3C Standards The 14th International World Wide Web Conference (WWW2005), ISBN 1-59593-051-5, pags. 1146-1147 Chiba, Japan, May 11-14, 2005

[17] Vicente Luque Centeno, Carlos Delgado Kloos, Jesús Arias Fisteus, Norberto Fernández García Estudio comparativo de herramientas de evaluación de la accesibilidad web V Jornadas de Ingeniería Telemática, JITEL 2005, ISBN 848408-346-2 Vigo, 12-14 de Septiembre de 2005 